\title{
Light-Front Hamiltonian and Path Integral Formulations of the Conformally Gauge-Fixed Polyakov D1 Brane Action
}

\author{
Usha Kulshreshtha ${ }^{1}$, Daya Shankar Kulshreshtha ${ }^{2}$ \\ ${ }^{1}$ Department of Physics, Kirori Mal College University of Delhi, Delhi, India \\ ${ }^{2}$ Department of Physics and Astrophysics University of Delhi, Delhi, India \\ E-mail: \{ushakulsh,dskulsh\}@gmail.com \\ Received January 6, 2011; revised March 25, 2011; accepted April 5, 2011
}

\begin{abstract}
In a recent paper we have studied the Hamiltonian and path integral quantizations of the conformally gauge-fixed Polyakov D1 brane action in the instant-form of dynamics using the equal world-sheet time framework on the hyperplanes defined by the world-sheet time $\sigma^{0}=\tau=$ constant. In the present work we quantize the same theory in the equal light-cone world-sheet time framework, on the hyperplanes of the light-front defined by the light-cone world-sheet time $\sigma^{+}=(\tau+\sigma)=$ constant, using the standard constraint quantization techniques in the Hamiltonian and path integral formulations. The light-front theory is seen to be a constrained system in the sense of Dirac, which is in contrast to the corresponding case of the instant-form theory, where the theory remains unconstrained in the sense of Dirac. The light-front theory is seen to possess a set of twenty six primary second-class contraints. In the present work Hamiltonian and path integral quantizations of this theory are studied on the light-front.
\end{abstract}

Keywords: Light-Front Quantization, Hamiltonian Quantization, Path Integral Quantization, Constrained Dynamics, Constraint Quantization, Gauge Symmetry, String Gauge Symmetry, String Theory, D-Brane Actions, Polyakov Action, Light-Cone Quantization.

\section{Introduction}

Polyakov D1 brane action [1-12] is one of the most widely studied topics in string theories [1-15]. The action possesses three well-known local gauge symmetries given by the two-dimensional world-sheet (WS) reparametrization invariance (WSRI) and the Weyl invariance (WI) [1-10]. When the action is considered under the conformal gauge-fixing it looses the above said string gauge symmetries as expected (owing to the conformal gauge-fixing). In a very recent paper [11,12], we have studied the conformally gauge-fixed Polyakov D1 brane action (CGFPD1BA) with and without a scalar dilaton field in the usual instant-form (IF) of dynamics, using the equal world-sheet (WS)-time (EWST) framework, on the hyperplanes defined by the WS-time $\sigma^{0}=\tau=$ constant [9-15]. The theory without a scalar dilaton field is seen to be an unconstrained system in the sense of Dirac [16], whereas in the presence of a scalar dilaton field it is seen to be a constrained system, possessing one primary and one secondary Gauss law constraint [9-15]. In the present work the same theory is studied on the light-front (LF) (in the front-form (FF) of dynamics) using the equal light-cone world-sheet time (ELCWST) framework on the hyperplanes of the LF defined by the light-cone world-sheet time $\sigma^{+}=(\tau+\sigma)=$ constant . The LF theory becomes a constrained system in the sense of Dirac (in contrast to the corresponding case of the IF theory [3]), and it is seen to possess a set of 26 primary second-class constraints. The LF theory is quantized using the standard constraint quantization techniques in the Hamiltonian and path integral formulations [9-21]. It is needless to say that the LF quantization (LFQ) has several distinct advantages over the usual IF quantization (IFQ) [22-27]. For a recent review on LFQ of field theories we refer to the work of Brodsky, Pauli and Pinsky [22-27]. In the next section we briefly recapitulate the IF theory $[11,12]$. In Section 3, we study the LFQ of the theory 
using the Hamiltonian and path integral formulations and finally the summary and discussion is given in Section 4 .

\section{Recapitulation of Instant-Form Theory}

In this section, we recapitulate very briefly the IF theory. The Polyakov D1 brane action describes the propagation of the D1 brane in a $d$-dimensional curved background $\boldsymbol{h}_{\alpha \beta}$ (with $d=10$ for the fermionic and $d=26$ for bosonic D1 brane) defined by [1-15]:

$$
\begin{gathered}
\tilde{S}=\int \mathcal{L} \mathrm{d}^{2} \sigma \\
\mathcal{L}=\left[-\frac{T}{2} \sqrt{-h} \boldsymbol{h}^{\alpha \beta} \boldsymbol{G}_{\alpha \beta}\right] ; h=\operatorname{det}\left(\boldsymbol{h}_{\alpha \beta}\right) \\
\boldsymbol{G}_{\alpha \beta}=\partial_{\alpha} X^{\mu} \partial_{\beta} X^{v} \eta_{\mu v} ; \boldsymbol{\eta}_{\mu v}=\operatorname{diag}(-1,+1, \cdots,+1) \\
\mu, v=0,1, \cdots,(d-1) ; \alpha, \beta=0,1
\end{gathered}
$$

Here $\sigma^{\alpha} \equiv(\tau, \sigma)$ are the two parameters describing the worldsheet (WS). The overdots and primes would denote the derivatives with respect to $\tau$ and $\sigma$.T is the string tension. $\boldsymbol{G}_{\alpha \beta}$ is the induced metric on the WS and $X^{\mu}(\tau, \sigma)$ are the maps of the WS into the $d$ dimensional Minkowski space and describe the strings evolution in space-time [1-10]. $\boldsymbol{h}_{\alpha \beta}$ are the auxiliary fields (which turn out to be proportional to the metric tensor $\boldsymbol{\eta}_{\alpha \beta}$ of the two-dimensional surface swept out by the string). One can think of $\tilde{S}$ as the action describing $d$ massless scalar fields $X^{\mu}$ in two dimensions moving on a background $\boldsymbol{h}_{\alpha \beta}$. Also because the metric components $\boldsymbol{h}_{\alpha \beta}$ are varied in the above equation, the 2dimensional gravitational field $\boldsymbol{h}_{\alpha \beta}$ is treated not as a given background field, but rather as an adjustable quantity coupled to the scalar fields [1-8]. The action $\tilde{S}$ possesses the well-known three local gauge symmetries given by the two-dimensional WS reparametrization invariance (WSRI) and the Weyl invariance (WI) [1-12]:

$$
\begin{aligned}
& X^{\mu} \rightarrow \tilde{X}^{\mu}=\left[X^{\mu}+\delta X^{\mu}\right] \\
& \delta X^{\mu}=\left[\zeta^{\alpha}\left(\partial_{\alpha} X^{\mu}\right)\right] \\
& \boldsymbol{h}^{\alpha \beta} \rightarrow \tilde{\boldsymbol{h}}^{\alpha \beta}=\left[\boldsymbol{h}^{\alpha \beta}+\delta \boldsymbol{h}^{\alpha \beta}\right] \\
& \delta \boldsymbol{h}^{\alpha \beta}=\left[\zeta^{\gamma} \partial_{\gamma} \boldsymbol{h}^{\alpha \beta}-\partial_{\gamma} \zeta^{\alpha} \boldsymbol{h}^{\gamma \beta}-\partial_{\gamma} \zeta^{\beta} \boldsymbol{h}^{\alpha \gamma}\right] \\
& \boldsymbol{h}_{\alpha \beta} \rightarrow\left[\Omega \boldsymbol{h}_{\alpha \beta}\right] ; \quad \Omega(\tau, \sigma)=\exp (2 \omega(\tau, \sigma))
\end{aligned}
$$

Here $\zeta^{\alpha}(\tau, \sigma)$ is a gauge parameter corresponding to the WSRI and $\Omega(\tau, \sigma)=\exp (2 \omega(\tau, \sigma))$ is a gauge parameter corresponding to the Weyl symmetry. The WSRI is defined by the first four equations involving the two gauge parameters $\zeta^{\alpha}$ and the WI is defined by the last equation and is specified by the gauge parameter $\Omega$ (or equivalently by $\omega$ ). Also the above theory being a gauge-invariant theory (possessing the local gauge symmetries including two WSRI and one WI symmetries), could be studied under approriate gauge-fixing the way one likes. However, one could also use the above three local gauge symmeties of the theory to choose $\boldsymbol{h}_{\alpha \beta}$ to be of a particular form [1-12], e.g., as follows:

$$
\boldsymbol{h}_{\alpha \beta}=\boldsymbol{\eta}_{\alpha \beta}=\left(\begin{array}{rr}
-1 & 0 \\
0 & +1
\end{array}\right)
$$

This is the so-called conformal gauge (CG). In this CG we have

$$
\sqrt{-h}=\sqrt{-\operatorname{det}\left(\boldsymbol{h}_{\alpha \beta}\right)}=+1
$$

and the action $\tilde{S}$ in this $\mathrm{CG}$ now becomes:

$$
\begin{aligned}
S_{1} & =\int \mathcal{L}_{1} \mathrm{~d}^{2} \sigma \\
\mathcal{L}_{1} & =\frac{-T}{2} \sqrt{-h} \boldsymbol{h}^{\alpha \beta} \boldsymbol{G}_{\alpha \beta} \\
& =\frac{-T}{2} \boldsymbol{\eta}^{\alpha \beta} \partial_{\alpha} X^{\mu} \partial_{\beta} X^{v} \boldsymbol{\eta}_{\mu v} \\
& =\frac{-T}{2} \partial^{\beta} X^{\mu} \partial_{\beta} X_{\mu} \\
& =\frac{-T}{2}\left[\partial_{\sigma} X^{\mu} \partial_{\sigma} X_{\mu}-\partial_{\tau} X^{\mu} \partial_{\tau} X_{\mu}\right] \\
& =\frac{-T}{2}\left[\left(X^{\prime}\right)^{2}-(\dot{X})^{2}\right] \\
\dot{X}^{\mu} & \equiv \frac{\partial X^{\mu}}{\partial \tau} \quad \text { and } \quad X^{\prime \mu}=\frac{\partial X^{\mu}}{\partial \sigma}
\end{aligned}
$$

This is the CGFPD1BA. The IFQ of this action has been studied by us recently in Ref. 3 and we recap it here very briefly. The canonical momenta conjugate to $X^{\mu}$ obtained from $L_{1}$ are:

$$
P^{\mu}:=\frac{\partial \mathcal{L}_{1}}{\partial\left(\partial_{\tau} X^{\mu}\right)}=T \partial_{\tau} X^{\mu}
$$

Here the velocities $\partial_{\tau} X^{\mu}=\frac{1}{T} P^{\mu}$ are expressible. The canonical Hamiltonian density corresponding to $\mathcal{L}_{1}$ is:

$$
\mathcal{H}_{1}^{c}=\left[P^{\mu}\left(\partial_{\tau} X_{\mu}\right)-L_{1}\right]=\left[\frac{1}{2 T} P^{2}+\frac{T}{2}\left(X^{\prime}\right)^{2}\right]
$$

The quantization of the system is trivial. The nonvanishing equal WS-time commutation relations for the theory described by $S_{1}$ are obtained as [1-4]:

$$
\left[X^{\mu}(\sigma, \tau), P_{v}\left(\sigma^{\prime}, \tau\right)\right]=i \delta_{v}^{\mu} \delta\left(\sigma-\sigma^{\prime}\right)
$$

where $\delta\left(\sigma-\sigma^{\prime}\right)$ is the one-dimensional Dirac distribution function. 
It is obvious from the above considerations that the above theory is unconstrained in the sense of Dirac. It may be important to emphasize here that an unconstrained system like the above theory is a gauge-noninvariant theory and is some what akin to a gauge-fixed gaugeinvariant theory which makes it a gauge-noninvariant system. In the presence of a scalar dilaton field the theory of course, becomes a constrained system in the sense of Dirac as shown in our earlier work [11,12]. For further details of the IF theory we refer to our earleir work of Ref. 11 and 12. In the next section, we study the LFQ of this theory [22-27].

\section{Light-Front Quantization}

In LFQ of the theory we use the three local gauge symmetries of the theory to choose $\boldsymbol{h}_{\alpha \beta}$ to be of a particular form as follows:

$$
\boldsymbol{h}_{\alpha \beta}:=\boldsymbol{\eta}_{\alpha \beta}=\left(\begin{array}{cc}
0 & -1 / 2 \\
-1 / 2 & 0
\end{array}\right)
$$

and

$$
\boldsymbol{h}^{\alpha \beta}:=\boldsymbol{\eta}^{\alpha \beta}=\left(\begin{array}{rr}
0 & -2 \\
-2 & 0
\end{array}\right)
$$

with

$$
\sqrt{-h}=\sqrt{-\operatorname{det}\left(\boldsymbol{h}_{\alpha \beta}\right)}=+1 / 2
$$

This is the so-called conformal gauge (CG) in the LFQ of the theory. Also, in the LFQ we use the LC variables defined by $[1-8]$ :

$$
\sigma^{ \pm}:=(\tau \pm \sigma) \text { and } X^{ \pm}:=\left(X^{0} \pm X^{1}\right) / \sqrt{2}
$$

The action $\tilde{S}$ in the above CG in the LFQ reads

$$
\begin{gathered}
S_{2}=\int L_{2} \mathrm{~d} \sigma^{+} \mathrm{d} \sigma^{-} \\
\mathcal{L}_{2}=\frac{-T}{2} \eta^{\alpha \beta} \partial_{\alpha} X^{\mu} \partial_{\beta} X^{v} \boldsymbol{\eta}_{\mu v} \\
=\frac{-T}{2} \partial^{\beta} X^{\mu} \partial_{\beta} X_{\mu} \\
=\left[\frac{-T}{2}\right]\left[\left(\partial_{+} X^{+}\right)\left(\partial_{-} X^{-}\right)+\left(\partial_{+} X^{-}\right)\left(\partial_{-} X^{+}\right)\right. \\
\left.+\left(\partial_{+} X^{i}\right)\left(\partial_{-} X^{i}\right)\right] \\
\mu, v=+,-, 2, \cdots,(d-1) ; i=2,3, \cdots,(d-1) .
\end{gathered}
$$

We now study the LFQ of the above Polyakov D1 brane action. The canonical momenta $P^{+}, P^{-}$and $P_{i}(i=2,3, \cdots, 25)$, obtained from the above equation are:

$$
P^{+}:=\frac{\partial \mathcal{L}_{2}}{\partial\left(\partial_{+} X^{-}\right)}=-\frac{T}{2}\left(\partial_{-} X^{+}\right)
$$

$$
\begin{gathered}
P^{-}:=\frac{\partial \mathcal{L}_{2}}{\partial\left(\partial_{+} X^{+}\right)}=-\frac{T}{2}\left(\partial_{-} X^{-}\right) \\
P_{i}:=\frac{\partial \mathcal{L}_{2}}{\partial\left(\partial_{+} X^{i}\right)}=-\frac{T}{2}\left(\partial_{-} X^{i}\right)
\end{gathered}
$$

The above equations, however, imply that the theory possesses twenty six primary constraints

$$
\begin{gathered}
\chi_{1}=\left(P^{+}+\frac{T}{2} \partial_{-} X^{+}\right) \approx 0 \\
\chi_{2}=\left(P^{-}+\frac{T}{2} \partial_{-} X^{-}\right) \approx 0 \\
\chi_{i}=\left(P_{i}+\frac{T}{2} \partial_{-} X^{i}\right) \approx 0, \quad i=2,3, \cdots, 25 .
\end{gathered}
$$

The canonical Hamiltonian density corresponding to $\mathcal{L}_{2}$ is

$$
\mathcal{H}_{2}^{c}=\left[P^{+}\left(\partial_{+} X^{-}\right)+P^{-}\left(\partial_{+} X^{+}\right)+P_{i}\left(\partial_{+} X^{i}\right)-\mathcal{L}_{2}\right] \approx 0
$$

After including the primary constraints $\chi_{i}$ in the canonical Hamiltonian density $\mathcal{H}_{2}^{c}$ with the help of Lagrange multiplier fields $u, v$ and $w_{i}$, the total Hamiltonian density $\mathcal{H}_{2}^{T}$ could be written as

$$
\begin{aligned}
\mathcal{H}_{2}^{T}= & {\left[u\left(P^{+}+\frac{T}{2} \partial_{-} X^{+}\right)+v\left(P^{-}+\frac{T}{2} \partial_{-} X^{-}\right)\right.} \\
& \left.+w_{i}\left(P_{i}+\frac{T}{2} \partial_{-} X^{i}\right)\right]
\end{aligned}
$$

We now treat $u, v$ and $w_{i}$ as dynamical. The Hamiltons equations obtained from the total Hamiltonian

$$
H_{2}^{T}=\int H_{2}^{T} \mathrm{~d} \sigma^{-}
$$

e.g., for the closed bosonic strings with periodic boundary conditions are now obtained as:

$$
\begin{aligned}
+\partial_{+} X^{-} & =\frac{\partial H_{2}^{T}}{\partial P^{+}}=u,-\partial_{+} P^{+}=\frac{\partial H_{2}^{T}}{\partial X^{-}}=\left(\frac{-T}{2} \partial_{-} v\right) \\
+\partial_{+} X^{+} & =\frac{\partial H_{2}^{T}}{\partial P^{-}}=v,-\partial_{+} P^{-}=\frac{\partial H_{2}^{T}}{\partial X^{+}}=\left(\frac{-T}{2} \partial_{-} u\right) \\
+\partial_{+} X^{i} & =\frac{\partial H_{2}^{T}}{\partial P_{i}}=w_{i},-\partial_{+} P_{i}=\frac{\partial H_{2}^{T}}{\partial X^{i}}=\left(\frac{-T}{2} \partial_{-} w_{i}\right) \\
+\partial_{+} u & =\frac{\partial H_{2}^{T}}{\partial p_{u}}=0,-\partial_{+} p_{u}=\frac{\partial H_{2}^{T}}{\partial u}=\left(P^{+}+\frac{T}{2} \partial_{-} X^{+}\right) \\
+\partial_{+} v & =\frac{\partial H_{2}^{T}}{\partial p_{v}}=0,-\partial_{+} p_{v}=\frac{\partial H_{2}^{T}}{\partial v}=\left(P^{-}+\frac{T}{2} \partial_{-} X^{-}\right) \\
+\partial_{+} w_{i} & =\frac{\partial H_{2}^{T}}{\partial p_{w_{i}}}=0,-\partial_{+} p_{w_{i}}=\frac{\partial H_{2}^{T}}{\partial w_{i}}=\left(P_{i}+\frac{T}{2} \partial_{-} X^{i}\right)
\end{aligned}
$$


These are the equations of motion of the theory that preserve the constraints of the theory in the course of time. Demanding that the primary constraints $\chi_{1}, \chi_{2}$ and $\chi_{i},(i=2,3, \cdots, 25)$, be preserved in the course of time one does not get any secondary constraints. The theory is thus seen to possess only twenty six constraints $\chi_{1}, \chi_{2}$ and $\chi_{i}$. The first-order Lagrangian density of the theory is

$$
\begin{aligned}
\mathcal{L}_{2}^{\mathrm{I} O}= & {\left[P^{+}\left(\partial_{+} X^{-}\right)+P^{-}\left(\partial_{+} X^{+}\right)+P_{i}\left(\partial_{+} X^{i}\right)\right.} \\
& \left.+p_{u}\left(\partial_{+} u\right)+p_{v}\left(\partial_{+} v\right)+p_{w_{i}}\left(\partial_{+} w_{i}\right)-\mathcal{H}_{2}^{T}\right] \\
= & {\left[\frac{-T}{2}\right]\left[u\left(\partial_{-} X^{+}\right)+v\left(\partial_{-} X^{-}\right)+w_{i}\left(\partial_{-} X^{i}\right)\right] }
\end{aligned}
$$

The matrix of the Poisson brackets of the constrains $\chi_{j}$ namely, $\boldsymbol{M}_{\alpha \beta}\left(\sigma, \sigma^{\prime}\right):=\left\{\chi_{\alpha}(\sigma), \chi_{\beta}\left(\sigma^{\prime}\right)\right\}_{\mathrm{P} B}$ is then calculated and the nonvanishing elements of this matrix are obtained as:

$$
\boldsymbol{M}_{12}=\boldsymbol{M}_{21}=\boldsymbol{M}_{i i}=T \partial_{-} \delta\left(\sigma-\sigma^{\prime}\right)
$$

The matrix $\boldsymbol{M}_{\alpha \beta}$ is seen to be nonsingular with the determinant given by

$$
\left[\left\|\operatorname{det}\left(\boldsymbol{M}_{\alpha \beta}\right)\right\|\right]^{1 / 2}=\left[T \partial_{-} \delta\left(\sigma-\sigma^{\prime}\right)\right]^{13}
$$

and the nonvanishing elements of the inverse of the matrix $\boldsymbol{M}_{\alpha \beta}$ (i.e. the elements of the matrix $\left.\left(\boldsymbol{M}^{-1}\right)_{\alpha \beta}\right)$ are obtained as:

$$
\left(M^{-1}\right)_{12}=\left(M^{-1}\right)_{21}=\left(M^{-1}\right)_{i i}=\frac{1}{2 T} \varepsilon\left(\sigma-\sigma^{\prime}\right)
$$

with

$$
\int M\left(\sigma, \sigma^{\prime \prime}\right) M^{-1}\left(\sigma^{\prime \prime}, \sigma^{\prime}\right) \mathrm{d} \sigma^{\prime \prime}=1_{26 \times 26} \delta\left(\sigma-\sigma^{\prime}\right)
$$

Here the step function $\varepsilon\left(\sigma-\sigma^{\prime}\right)$ is defined as

$$
\varepsilon\left(\sigma-\sigma^{\prime}\right):= \begin{cases}+1 & \text { for }\left(\sigma-\sigma^{\prime}\right)>0 \\ -1 & \text { for }\left(\sigma-\sigma^{\prime}\right)<0\end{cases}
$$

Now, following the standard Dirac quantization procedure in the Hamiltonian formulation [5], the nonvanishing equal light-cone world-sheet time (ELCWST) ( $\sigma^{+}=\sigma^{++}=\tau=$ constant ) commutators of the theory describing the Polyakov D1 brane action $S^{N}$ are finally obtained as [9-21]:

$$
\begin{aligned}
& {\left[X^{+}\left(\sigma^{-}, \sigma^{+}\right), P^{-}\left(\sigma^{\prime^{-}}, \sigma^{+}\right)\right]=\frac{i}{2} \delta\left(\sigma^{-}-\sigma^{\prime^{-}}\right)} \\
& {\left[X^{-}\left(\sigma^{-}, \sigma^{+}\right), P^{+}\left(\sigma^{\prime-}, \sigma^{+}\right)\right]=\frac{i}{2} \delta\left(\sigma^{-}-\sigma^{\prime-}\right)} \\
& {\left[X^{i}\left(\sigma^{-}, \sigma^{+}\right), P_{i}\left(\sigma^{\prime-}, \sigma^{+}\right)\right]=\frac{i}{2} \delta\left(\sigma^{-}-\sigma^{\prime-}\right)}
\end{aligned}
$$

$$
\begin{aligned}
& {\left[X^{-}\left(\sigma^{-}, \sigma^{+}\right), X^{+}\left(\sigma^{\prime-}, \sigma^{+}\right)\right]=\frac{i}{2 T} \varepsilon\left(\sigma^{-}-\sigma^{\prime-}\right)} \\
& {\left[X^{i}\left(\sigma^{-}, \sigma^{+}\right), X^{i}\left(\sigma^{\prime-}, \sigma^{+}\right)\right]=\frac{i}{2 T} \varepsilon\left(\sigma^{-}-\sigma^{\prime}\right)} \\
& {\left[P^{+}\left(\sigma^{-}, \sigma^{+}\right), P^{-}\left(\sigma^{-}, \sigma^{+}\right)\right]=\frac{-i T}{4} \partial_{-} \delta\left(\sigma^{-}-\sigma^{\prime-}\right)} \\
& {\left[P_{i}\left(\sigma^{-}, \sigma^{+}\right), P_{i}\left(\sigma^{\prime-}, \sigma^{+}\right)\right]=\frac{-i T}{4} \partial_{-} \delta\left(\sigma^{-}-\sigma^{\prime-}\right)}
\end{aligned}
$$

In the path integral formulation, the transition to the quantum theory, is, however, made by writing the vacuum to vacuum transition amplitude called the generating functional $Z_{2}\left[J_{k}\right]$ of the theory in the presence of external sources $J_{k}$ as follows [9-21]:

$$
\begin{aligned}
& Z_{2}\left[J_{k}\right]:=\int[\mathrm{d} \mu] \exp \left[i \int \mathrm{d} \sigma^{+} \mathrm{d} \sigma^{-}\left(L_{2}^{I O}+J_{k} \Phi^{k}\right)\right] \\
& =\int[\mathrm{d} \mu] \exp \left[i \int \mathrm { d } \sigma ^ { + } \mathrm { d } \sigma ^ { - } \left(P^{+} \partial_{+} X^{-}+P^{-} \partial_{+} X^{+}+P_{i} \partial_{+} X^{i}\right.\right. \\
& \left.\left.+p_{u} \partial_{+} u+p_{v} \partial_{+} v+p_{w_{i}} \partial_{+} w_{i}-H_{2}^{T}+J_{k} \Phi^{k}\right)\right] \\
& =\int[\mathrm{d} \mu] \exp \left[i \int \mathrm { d } \sigma ^ { + } \mathrm { d } \sigma ^ { - } \left[\frac { - T } { 2 } \left(u \partial_{-} X^{+}\right.\right.\right. \\
& \left.\left.\left.+v \partial_{-} X^{-}+w_{i} \partial_{-} X^{i}\right)+J_{k} \Phi^{k}\right]\right]
\end{aligned}
$$

where the phase space variables of the theory are $\Phi^{k} \equiv\left(X^{+}, X^{-}, X^{i}, u, v, w_{i}\right)$ with the corresponding respective canonical conjugate momenta:

$\Pi_{k} \equiv\left(P^{-}, P^{+}, P_{i}, p_{u}, p_{v}, p_{w_{i}}\right)$. The functional measure $[\mathrm{d} \mu]$ of the generating functional $Z_{2}\left[J_{k}\right]$ is obtained as [9-21]:

$$
\begin{aligned}
& {[\mathrm{d} \mu]=\left[T \partial_{-} \delta\left(\sigma^{-}-\sigma^{--}\right)\right]^{13}\left[\mathrm{~d} X^{+}\right]\left[\mathrm{d} X^{-}\right][\mathrm{d} u][\mathrm{d} v]} \\
& {\left[\mathrm{d} P^{-}\right]\left[\mathrm{d} P^{+}\right]\left[\mathrm{d} p_{u}\right]\left[\mathrm{d} p_{v}\right] \Pi\left(\left[\mathrm{d} X^{i}\right]\left[\mathrm{d} P_{i}\right]\left[\mathrm{d} w_{i}\right]\left[\mathrm{d} p_{w_{i}}\right]\right)} \\
& \delta\left[\left(P^{+}+\frac{T}{2} \partial_{-} X^{+}\right) \approx 0\right] \delta\left[\left(P^{-}+\frac{T}{2} \partial_{-} X^{-}\right) \approx 0\right] \\
& \Pi\left(\delta\left[\left(P_{i}+\frac{T}{2} \partial_{-} X^{i}\right) \approx 0\right]\right) .
\end{aligned}
$$

The LF Hamiltonian and path integral quantizations of our theory described by the Polyakov D1 brane action $S_{2}$ are now complete.

\section{Summary and Discussion}

Polyakov D1 brane action [1-12] possesses three wellknown local gauge symmetries given by the two-dimensional world-sheet reparametrization invariance and the Weyl invariance [1-10]. When the action is considered under the conformal gauge-fixing it looses the above said 
string gauge symmetries as expected. In a very recent paper $[11,12]$, we have studied the conformally gaugefixed Polyakov D1 brane action with and without a scalar dilaton field in the usual instant-form (IF) of dynamics, using the equal world-sheet-time framework, on the hyperplanes defined by the WS-time $\sigma^{0}=\tau=$ constant [1-21]. The theory without a scalar dilaton field is seen to be an unconstrained system in the sense of Dirac [16], whereas in the presence of a scalar dilaton field it is seen to be a constrained system, possessing one primary and one secondary Gauss law constraint [9-21].

In the present work the same theory is studied on the LF (using the front-form of dynamics) in the equal lightcone world-sheet time (ELCWST) framework on the hyperplanes of the LF defined by the light-cone worldsheet time $\sigma^{+}=(\tau+\sigma)=$ constant [9,10,22-27]. The LF theory is seen to become a constrained system in the sense of Dirac (which is in contrast to the corresponding case of the IF theory $[11,12])$, and it is seen to possess a set of 26 primary second-class constraints.

It may be worthwhile to record a few important points here. The unconstrained IF theory as mentioned above is in fact analogues to the usual Klein-Gordon field theory in three-space one-time dimension which is an unconstrained theory in the sense of Dirac. This in turn is equivalent to a gauge-noninvariant or equivalently a gaugefixed theory which does not have a gauge symmetry (owing to the gauge-fixing). The corresponding LF theory is on the other hand is a constrained system in the sense of Dirac as seen in Section 3, where it has been explicitly shown to be a constrained system possessing a set of 26 second-class constraints. This theory could then we used to construct an equivalent gauge-invariant theory using the techniques of constrained dynamics (albeit constraint quantization) which is however, outside the scope of the present work. The same may however, not be true of the corresponding unconstrained IF theory.

The LF theory is quantized using the standard constraint quantization techniques in the Hamiltonian and path integral formulations. It is needless to say that the LF quantization (LFQ) has undisputedly several distinct advantages [6] over the usual IF quantization (IFQ) [22-27]. One of the most important advantages of the LF framework e.g., is that the LF theory provides the largest number of kinematical generators of the Poincare transformations in Hamiltonian dynamics. For a recent review on LFQ of field theories we refer to the work of Brodsky, Pauli and Pinsky [22-27].

Also, we like to make a few comments about further solving the LF theory. It is possible to write down the solutions of the LF theory on the reduced hypersurface of the constraints of the theory where one implements the constraints of the theory strongly and this could be achieved in the Hamiltonian as well as in the path integral formulation of the theory. This is however, outside the scope of the present work. If one tryies to do a similar thing with the IF theory then that would simply not be possible because even though the IF theory is gaugenoninvariant and is equivalent to a gauge-fixed gaugeinvariant theory but one simply can not do any of the manipulations which could be done within the acceptable framework of the techniques of constraint quantization simply because (the IF theory) does not have any constraint structure. One well known examples of this concerns the Batalin-Fradkin-Vilkovisky quantization of a gauge-noninvariant theory where one enlarges the phase space of a classical theory or the Hilbert space of the corresponding quantum theory by introducing some additional fields in to the theory by modifying the secondclass constraints of the theory in such a manner that each of the second-class constraint of the theory becomes a first-class constraint. This in principle, could be done with the LF theory because its constraint structure is known, but it would simply be impossible to do such a thing with the IF theory for which the constraint structure is not known.

Further, in the canonical quantization of the theory while going from ELCWST Dirac brackets of the theory to the corresponding ELCWST commutation relations one could encounter the problem of operator ordering [28] because the product of canoncial variable of the theory are involved in the classical description of the theory e.g., in the calculation of the Dirac brackets. These variables are envisaged as noncommuting operators in the quantized theory leading to the problem of so-called operator ordering [28]. This problem could, however, be resolved [28] by demanding that all the string fields and momenta of the theory are Hermitian operators and that all the canoncial commutation relations be con- sistent with the Hermiticity of the operators [28].

Also it is important to mention here that in our work we have not imposed any boundary conditions for the open and closed strings separately. There are two ways to take these boundary conditions into account: (a) one way is to impose them directly in the usual way for the open and closed strings separately in an appropriate manner [1-10], (b) an alternative second way is to treat these boundary conditions as the Dirac primary constraints [29] and study the theory accordingly [29].

\section{References}

[1] D. Luest and S. Theisen, "Lectures on String Theory," Lecture Notes in Physics, Vol. 346, Springer Verlag, Berlin, 1989.

[2] L. Brink and M. Henneaux, "Principles of String Theory," Plenum Press, New York, 1988. 
[3] C. V. Johnson, "D-Brane Primer," hep-th/0007170.

[4] M. Aganagic, J. Park, C. Popescu and J. Schwarz, "Dual D-Brane Actions," Nuclear Physics B, Vol. 496, No. 1-2, 1997, pp. 215-230. doi:10.1016/S0550-3213(97)00257-5

[5] M. A. Zeid and C. M. Hull, "Intrinsic Geometry of D-Branes," Physics Letters B, Vol. 404, No. 3-4, 1997, pp. 264-270. doi:10.1016/S0370-2693(97)00570-4

[6] C. Schmidhuber, "D-Brane Actions," Nuclear Physics B, Vol. 467, No. 1-2, 1996, pp. 146-158. doi:10.1016/0550-3213(96)00092-2

[7] S. P. de Alwis and K. Sato, "D-Strings and F-Strings from String Loops," Physical Review D, Vol. 53, No. 12, 1996, pp. 7187-7196. doi:10.1103/PhysRevD.53.7187

[8] A. A. Tseytlin, "Self Duality of Born-Infeld Action and Dirichlet Three-Brane of Type IIB Super String Theory," Nuclear Physics B, Vol. 469, No. 1-2, 1996, pp. 51-67. doi:10.1016/0550-3213(96)00173-3

[9] U. kulshreshtha and D. S. Kulshreshtha, "Conformally Gauge-Fixed Polyakov $\mathrm{D}_{1}$-Brane Action in the Presence of a 2-Form Gauge Field: The Instant-Form and FrontForm Hamiltonian and Path Integral Formulations," Physics Letters B, Vol. 555, No. 3-4, 2003, pp. 255-263. doi:10.1016/S0370-2693(03)00056-X

[10] D. S. Kulshreshtha, "Polyakov D1 Brane Action on the Light-Front," Invited Talk at the Light-Cone 2008: Relativistic Nuclear and Particle Physics (2008), Mulhouse, 7-11 July 2008, Published in PoS LC2008: 007, 2008, hep-th/0809.1038.

[11] U. Kulshreshtha and D. S. Kulshreshtha, "Hamiltonian and Path Integral Quantization of the Conformally Gauge-Fixed Polyakov D1 Brane Action in the Presence of a Scalar Dilation Field," International Journal of Theoretical Physics, Vol. 48, No. 4, 2009, pp. 937-944. doi:10.1007/s10773-008-9866-Z

[12] D. S. Kulshreshtha, "Light-Front Quantization of the Polyakov D1 Brane Action with a Scalar Dilaton Field," Invited Talk at the Light-Cone 2007: Relativistic Hadronic and Nuclear Physics (LC2007), Columbus, 14-18 May 2007, hep-th/0711.1342.

[13] U. Kulshreshtha and D. S. Kulshreshtha, "Hamiltonian and Path Integral Formulations of the Dirac-Born-Infeld-Nambu-Goto D1 Brane Action with and without a Dilaton Field under Gauge-Fixing," European Physical Journal C, Vol. 29, No.3, 2003, pp. 453-461. doi:10.1140/epjc/s2003-01239-8

[14] U. Kulshreshtha and D. S. Kulshreshtha, "Hamiltonian and Path Integral Formulations of the Nambu-Goto D1 Brane Action with and without a Dilaton Field under Gauge-Fixing," International Journal of Theoretical Physics, Vol. 43, No. 12, 2004, pp. 2355-2369. doi:10.1007/s10773-004-7704-5

[15] U. Kulshreshtha and D. S. Kulshreshtha, "Hamiltonian and Path Integral Formulations of the Born-InfeldNambu-Goto D1 Brane Action with and without a Dilaton Field under Gauge-Fixing," International Journal of Theoretical Physics, Vol. 44, No. 5, 2005, pp. 587-603. doi:10.1007/s10773-005-3985-6

[16] P. A. M. Dirac, "Generalized Hamiltonian Dynamics,"
Canadian Journal of Mathematics, Vol. 2, 1950, pp. 129-148. doi:10.4153/CJM-1950-012-1

[17] M. Henneaux and C. Teitleboim, "Quantization of Gauge Systems," Princeton University Press, New Jersey, 1992.

[18] P. Senjanovic, "Path Integral Quantization of Field Theories with Second-Class Constraints," Annals of Physics, Vol. 100, No. 1-2, 1976, pp. 227-261. Erratum: Annals of Physics, Vol. 209, No. 1, 1991, p. 248.

[19] U. Kulshreshtha, "Hamiltonian, Path Integral and BRST Formulations of the Chern-Simons-Higgs Theory in the Broken Symmetry Phase," Physica Scripta, Vol. 75, No. 6, 2007, pp. 795-802. doi:10.1088/0031-8949/75/6/009

[20] U. Kulshreshtha, "Vector Scwinger Model with a Photon Mass Term: Gauge-Invariant Reformulation, Operator Solutions and Hamiltonian and Path Integral Formulations," Modern Physics Letters A, Vol. 22, No. 39, 2007, pp. 2993-3001. doi:10.1142/S0217732307023663

[21] U. Kulshreshtha and D. S. Kulshreshtha, "Gauge-Invariant Reformulation of the Vector Schwinger Model with a Photon Mass Term and Its Hamiltonian, Path Integral and BRST Formulations," International Journal of Modern Physics A, Vol. 22, No. 32, 2007, pp. 6183-6201. doi:10.1142/S0217751X07038049

[22] P. A. M. Dirac, "Forms of Relativistic Dynamics," Reviews of Modern Physics, Vol. 21, No. 3, 1949, pp. 392-399. doi:10.1103/RevModPhys.21.392

[23] S. J. Brodsky, H. C. Pauli and S. S. Pinsky, "Quantum Chromodynamics and Other Field Theories on the LightCone," Physics Reports, Vol. 301, No. 4-6, 1998, pp. 299-486. doi:10.1016/S0370-1573(97)00089-6

[24] U. Kulshreshtha, "Hamiltonian and BRST Formulations of the Nelsen-Olesen Model," International Journal of Theoretical Physics, Vol. 41, No. 2, 2002, pp. 273-291. doi:10.1023/A:1014058806710

[25] U. Kulshreshtha, "Light-Front Hamiltonian, Path Integral and BRST Formulations of the Nelsen-Olsen (Bogomol'nyi) Model in the Light-Cone Gauges," International Journal of Theoretical Physics, Vol. 46, No. 10, 2007, pp. 2516-2530. doi:10.1007/s10773-007-9367-5

[26] U. Kulshreshtha, D. S. Kulshreshtha and J. P. Vary, "Light-Front Hamiltonian, Path Integral and BRST Formulations of the Chern-Simons-Higgs Theory under Appropriate Gauge-Fixing," Physics Scripta, Vol. 82, No. 5, 2010, p. 055101. doi:10.1088/0031-8949/82/05/055101

[27] U. Kulshreshtha, D. S. Kulshreshtha and J. P. Vary, "LightFront Hamiltonian, Path Integral and BRST Formulations of the Chern-Simons Theory under Appropriate GaugeFixing," Journal of Modern Physics, Vol. 1, No. 6, 2010 , pp. 385-392. doi:10.4236/jmp.2010.16055

[28] J. Maharana, "Quantization of Nonlinear Sigma Model in Constrained Hamiltonian Formalism," Physics Letters B, Vol. 128, No. 6, 1983, pp. 411-414. doi:10.1016/0370-2693(83)90928-0

[29] M. M. Sheikh-Jabbari and A. Shirzad, "Boundary Conditions as Dirac Constraints," European Physical Journal C, Vol. 19, No. 2, 2001, pp. 383-390. doi:10.1007/s100520100590 